\title{
Bilateral Subdural Haematoma and CPAP Use: A Possible Association
}

\author{
Beatrice Khater, Vicky Kassouf, Georges Haddad, Roula Hourani \\ American University of Beirut, Beirut, Lebanon
}

Received: $16 / 03 / 2020$

Accepted: 07/04/2020

Published: $28 / 05 / 2020$

How to cite this article: Khater B, Kassouf V, Haddad G, Hourani R. Bilateral subdural haematoma and CPAP use: a possible association. EJCRIM 2020;7: doi:10.12890/2020_001602.

Conflicts of Interests: The Authors declare that there are no competing interests.

This article is licensed under a Commons Attribution Non-Commercial 4.0 License

\section{ABSTRACT}

Obstructive sleep apnoea (OSA) is a common condition usually treated with continuous positive airway pressure (CPAP). No reports have linked it to an acute subdural haematoma. A 54-year-old white man who had hypertension well controlled with an angiotensin II receptor blocker, presented with a 2-week history of occipital headache with no other focal neurological symptoms. The headache began 12 days after he had started using CPAP for OSA. A brain MRI performed 2 weeks later showed bilateral subdural haematomas which were chronic on the left and sub-acute/acute on the right. Since the patient was clinically stable with no focal neurological deficits, he received prednisone for 3 weeks and was followed up with consecutive CT scans demonstrating gradual regression of the haematomas. This is the first report showing that subdural haematomas could be linked to CPAP use.

\section{LEARNING POINTS}

- Primary care physicians, pulmonologists and neurologists should be alert for unexplained headache in a patient on continuous positive airway pressure (CPAP).

- Subdural haematoma may be a rare reported side effect of CPAP use.

- Subdural haematoma in a stable patient with no focal neurological deficits can be treated conservatively with close monitoring and follow-up.

\section{KEYWORDS}

Headache, subdural haematoma, obstructive sleep apnoea, CPAP

\section{CASE PRESENTATION}

A 54-year-old white man, who worked as a consultant and spent long hours in front of a screen, presented with a 2-week history of headache. He had hypertension controlled with an angiotensin II receptor blocker. His BMI was $28 \mathrm{~kg} / \mathrm{m}^{2}$. He described his headache as heaviness in the occipital area, associated with a foggy sensation and noise sensitivity. He denied any vision changes, weakness or focal neurological symptoms. There was no history of head trauma or strenuous exercise, or of smoking or use of recreational drugs. There was no use of aspirin or any other NSAID for pain control. There was no personal or family history of coagulopathy. The patient reported that the headache had started 12 days after he started using continuous positive airway pressure (CPAP) at night for obstructive sleep apnoea (OSA).

The physical examination, including vital signs and a full neurological investigation, was normal.

\section{Methods and Procedures}

In view of the new onset headache in a patient over 50 years of age, an MRI of the brain without contrast was requested. It showed bilateral subdural collections: a $0.7 \mathrm{~cm}$ left chronic subdural haematoma (SDH), and a $1.4 \mathrm{~cm}$ right sub-acute/acute haematoma, with effacement of 
the peri-mesencephalic and suprasellar cisterns (Fig. 1). Investigations for blood coagulation disorders were all negative. A CT scan on day 7 showed stable haematomas, and on day 20 showed persistence of the SDH with a slight decrease in the thickness of the left collection. On day 30, a full spine MRI with contrast ruled out cerebrospinal fluid (CSF) leak.

Since the patient was stable, he was discharged on prednisone $(0.5 \mathrm{mg} / \mathrm{kg})$ for 2 weeks with close clinical monitoring. His symptoms resolved gradually within 10 days. A brain CT performed 4 months after cessation of CPAP use showed complete resolution of the haematomas (Fig. 2), while a brain MRI carried out 30 months later showed no new lesions (Fig. 2). The patient refused to use CPAP again.
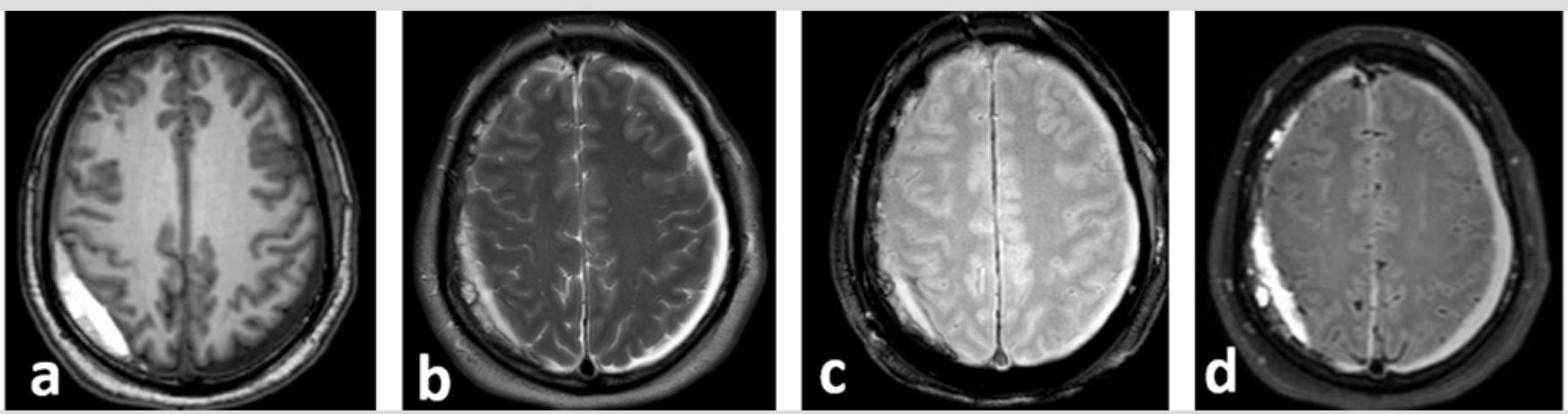

Figure 1. Brain MRI at presentation: axial T1 weighted image (WI) (a), axial T2-WI (b), T2* (c) and fluid attenuated inversion recovery image (FLAIR) (d). There are bilateral subdural collections. The left subdural haematoma (SDH) measures $0.7 \mathrm{~cm}$ in thickness, and is hypointense on T1 and hyperintense on T2, indicating a chronic subdural haematoma. The right SDH measures $1.4 \mathrm{~cm}$ in thickness and shows a high signal on T1-WI and a mixed hyper- and hypo-signal on T2-WI, suggestive of subacute/acute haematoma.
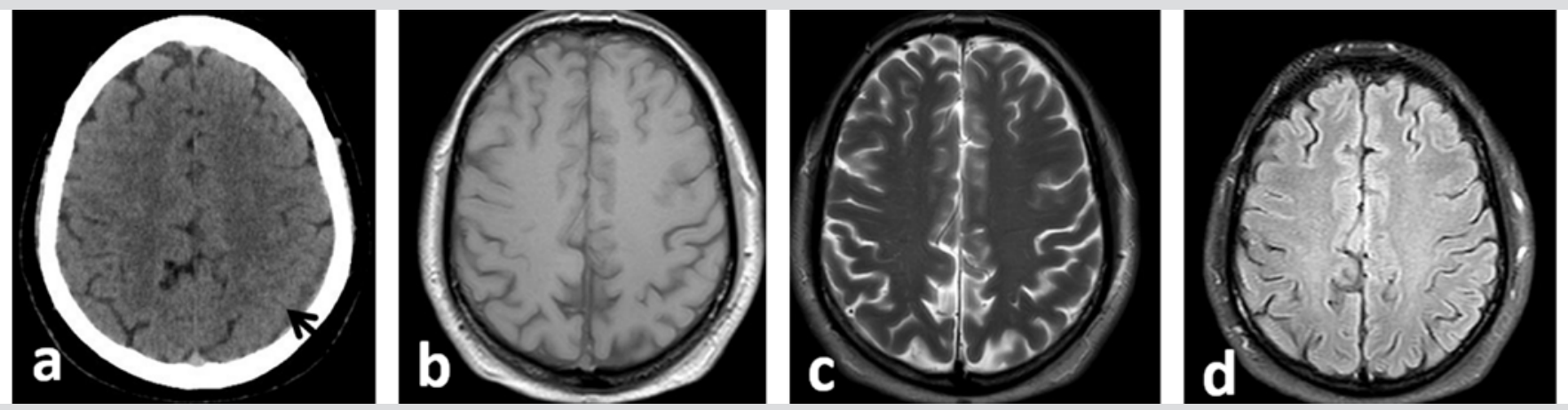

Figure 2. (a) Axial non-enhanced CT scan of the brain: follow-up CT scan of the brain after 4 months at the same level as Fig. 1 demonstrates complete resolution of the right subdural haematoma (SDH) and a decrease in the size of the left SDH (black arrow). Follow-up brain MRI after 30 months: axial T1-WI (b), T2-WI (c) and FLAIR images (d) show complete resolution of the bilateral subdural collections

\section{DISCUSSION}

CPAP is a first-line treatment for patients with OSA and an adjunctive therapy for hypertension in patients with both conditions ${ }^{[1]}$. It is commonly used due to its safety profile and beneficial effects on quality of life ${ }^{[2]}$, including but not limited to improvement of headaches ${ }^{[3]}$ and neurocognitive recovery ${ }^{[4,5]}$. Its use occasionally results in minor deleterious side effects including dry mouth, skin irritation, and sleep and metabolic disturbances ${ }^{[6,7]}$. To date, no major side effects from CPAP use have been reported in the literature and no study has connected it to SDH. This is the first report showing that SDH could be linked to CPAP use, although this association could be coincidental.

$\mathrm{SDH}$ is a collection of blood secondary to tearing of the bridging veins between the arachnoid layer and the dura. It can be idiopathic or can develop secondary to coagulopathies, the consumption of anticoagulants or antiplatelet drugs, trauma or intracranial hypotension [8]. The absence of direct trauma to our patient's head and the lack of anticoagulant intake excluded traumatic and iatrogenic causes. Moreover, haematological investigations ruled out blood coagulopathies.

The age of the SDH found on the first images coincided with the start of CPAP use. They were assumed to be more than 21 days old, and our patient had started using CPAP 26 days before undergoing the first brain MRI.

As regards intracranial pressure, our patient had never had brain or spine surgery or a lumbar puncture, nor did he ever have any symptoms of 
CSF rhinorrhoea that might cause intracranial hypotension. Nevertheless, a full spine MRI with contrast was performed to rule out possible CSF leak but none was found. At this stage the only possible cause for the SDH in this patient was spontaneous low intracranial pressure. Review of our patient's history indicated that the only change in his lifestyle was the use of CPAP at night for OSA, 12 days before the headache started. Studies have shown that CPAP use can provoke changes to CSF flow dynamics as it increases the CSF response to hypoxia in patients with OSA ${ }^{[9,10]}$. However, to date, no study has found a direct association between CPAP use and SDH. However, such changes have been investigated in cats ${ }^{[11]}$, and in multiple healthy volunteers ${ }^{[9,10,12]}$. Results have shown that the use of CPAP at $10-15$ mmHg generates high intra-abdominal pressure that is transmitted to the craniospinal space and leads to an increase in intracranial pressure (ICP) and a subsequent decrease in the cerebral perfusion pressure.

Therefore a possible mechanism linking CPAP to SDH could be that increased ICP caused by CPAP use is followed by a rebound decrease in ICP thus leading to bilateral SDH. However, we cannot rule out that the association between SDH and CPAP use is coincidental. Further cases should be reported in order to clearly identify this association.

\section{REFERENCES}

1. Epstein LJ, Kristo D, Strollo PJ Jr, Friedman N, Malhotra A, Patil SP, et al.; Adult Obstructive Sleep Apnea Task Force of the American Academy of Sleep Medicine. Clinical guideline for the evaluation, management and long-term care of obstructive sleep apnea in adults. J Clin Sleep Med 2009;5(3):263-276.

2. Jing J, Huang T, Cui W, Shen H. Effect on quality of life of continuous positive airway pressure in patients with obstructive sleep apnea syndrome: a meta-analysis. Lung 2008;186(3):131-144.

3. Johnson KG, Ziemba AM, Garb JL. Improvement in headaches with continuous positive airway pressure for obstructive sleep apnea: a retrospective analysis. Headache 2013;53(2):333-343.

4. Rosenzweig I, Glasser M, Crum WR, Kempton MJ, Milosevic M, McMillan A, et al. Changes in neurocognitive architecture in patients with obstructive sleep apnea treated with continuous positive airway pressure. EBioMedicine 2016;7:221-229.

5. Brill A-K, Horvath T, Seiler A, Camilo M, Haynes AG, Ott SR, et al. CPAP as treatment of sleep apnea after stroke: a meta-analysis of randomized trials. Neurology 2018;90(14):e1222-e1230.

6. Ulander M, Johansson MS, Ewaldh AE, Svanborg E, Broström A. Side effects to continuous positive airway pressure treatment for obstructive sleep apnoea: changes over time and association to adherence. Sleep Breath 2014;18(4):799-807.

7. Shechter A. Effects of continuous positive airway pressure on energy balance regulation: a systematic review. Eur Respir J 2016;48(6):1640-1657.

8. Yadav YR, Parihar V, Namdev H, Bajaj J. Chronic subdural hematoma. Asian J Neurosurg 2016;11(4):330-342.

9. Yiallourou TI, Schmid Daners M, Kurtcuoglu V, Haba-Rubio J, Heinzer R, Fornari E, et al. Continuous positive airway pressure alters cranial blood flow and cerebrospinal fluid dynamics at the craniovertebral junction. Interdiscip Neurosurg 2015;2(3):152-159.

10. Foster GE, Hanly PJ, Ostrowski M, Poulin MJ. Effects of continuous positive airway pressure on cerebral vascular response to hypoxia in patients with obstructive sleep apnea. Am J Respir Crit Care Med 2007;175(7):720-725.

11. Aidinis SJ, Lafferty J, Shapiro HM. Intracranial responses to PEEP. Anesthesiology 1976;45(3):275-286.

12. Hörmann C, Mohsenipour I, Gottardis M, Benzer A. Response of cerebrospinal fluid pressure to continuous positive airway pressure in volunteers. Anesth Analg 1994;78(1):5457. 